\title{
Quantum Integrals for a Semi-Infinite $q$-Boson System with Boundary Interactions ${ }^{\star}$
}

\author{
Jan Felipe VAN DIEJEN ${ }^{\dagger}$ and Erdal EMSIZ $\ddagger$ \\ † Instituto de Matemática y Física, Universidad de Talca, Casilla 747, Talca, Chile \\ E-mail: diejen@inst-mat.utalca.cl \\ ¥ Facultad de Matemáticas, Pontificia Universidad Católica de Chile, \\ Casilla 306, Correo 22, Santiago, Chile \\ E-mail: eemsiz@mat.puc.cl
}

Received February 04, 2015, in final form April 30, 2015; Published online May 06, 2015

http://dx.doi.org/10.3842/SIGMA.2015.037

\begin{abstract}
We provide explicit formulas for the quantum integrals of a semi-infinite $q$-boson system with boundary interactions. These operators and their commutativity are deduced from the Pieri formulas for a $q \rightarrow 0$ Hall-Littlewood type degeneration of the MacdonaldKoornwinder polynomials.
\end{abstract}

Key words: q-bosons; boundary interactions; Hall-Littlewood functions; hyperoctahedral symmetry; Pieri formulas; integrability

2010 Mathematics Subject Classification: 33D52; 81R50; 81T25; 82B23

\section{Introduction}

The $q$-boson system $[1,24]$ constitutes an integrable $q$-deformed lattice regularization of the quantum nonlinear Schrödinger equation $[11,15,17]$ built of $q$-oscillators $[12,20]$. Its $n$-particle Bethe Ansatz eigenfunctions amount to the celebrated Hall-Littlewood functions [7, 16, 26]. The model in question can moreover be viewed as a degeneration of the recently found stochastic $q$-Hahn particle system [2, 21, 25].

By deforming the $q$-oscillator algebra at the boundary, a semi-infinite $q$-boson system was constructed [6, 8] with eigenfunctions given by the hyperoctahedral Hall-Littlewood functions $[18,27]$ that arise as a $(q \rightarrow 0)$ limit of the Macdonald-Koorwinder polynomials [14, 18]. In the present note, we use a corresponding $q \rightarrow 0$ degeneration of the Pieri formulas for the Macdonald-Koornwinder polynomials [3] to arrive at explicit formulas for the commuting quantum integrals of the latter semi-infinite $q$-boson system with boundary interactions.

For the $q$-boson systems on the finite periodic lattice and on the (bi-)infinite lattice, analogous descriptions of the commuting quantum integrals stemming from the Pieri formulas for the Hall-Littlewood functions can be found in [5, 16, 26] and in [7], respectively. Previously, Pieri formulas for Macdonald's ( $q$-deformed Hall-Littlewood) polynomials were interpreted in a similar vein as eigenvalue equations for the quantum integrals of lattice Ruijsenaars-Schneider type models $[4,10,22,23]$.

\section{Commuting quantum integrals}

Let $e_{1}, \ldots, e_{n}$ be the standard unit basis of $\mathbb{R}^{n}$ and let $\Lambda$ denote the cone of integer partitions $\lambda=\left(\lambda_{1}, \ldots, \lambda_{n}\right)$ with parts $\lambda_{1} \geq \cdots \geq \lambda_{n} \geq 0$.

\footnotetext{
${ }^{\star}$ This paper is a contribution to the Special Issue on Exact Solvability and Symmetry Avatars in honour of Luc Vinet. The full collection is available at http://www.emis.de/journals/SIGMA/ESSA2014.html
} 
For $l \in\{1, \ldots, n\}$, we define the following difference operator $H_{l}$ acting on the space $C(\Lambda)$ of complex lattice functions $f: \Lambda \rightarrow \mathbb{C}$ :

$$
\left(H_{l} f\right)(\lambda):=\sum_{\substack{J_{+}, J_{-} \subset\{1, \ldots, n\} \\ J_{+} \cap J_{-}=\varnothing,\left|J_{+}\right|+\left|J_{-}\right| \leq l \\ \lambda+e_{J_{+}}-e_{J_{-}} \in \Lambda}} U_{J_{+}^{c} \cap J_{-}^{c}, l-\left|J_{+}\right|-\left|J_{-}\right|}(\lambda) W_{J_{+}, J_{-}}(\lambda) f\left(\lambda+e_{J_{+}}-e_{J_{-}}\right),
$$

where $|J|$ refers to the cardinality of $J \subset\{1, \ldots, n\}, J^{c}:=\{1, \ldots, n\} \backslash J$, and $e_{J}:=\sum_{j \in J} e_{j}$. The coefficients of the difference operator in question are built of the factors

$$
W_{J_{+}, J_{-}}(\lambda):=\prod_{\substack{j \in J_{+} \\ \lambda_{j}=0}}\left(\prod_{0 \leq r<s \leq 2}\left(1-t_{r} t_{s} t^{n-j}\right)\right) \prod_{\substack{1 \leq j<k \leq n \\ \lambda_{j}=\lambda_{k}, \epsilon_{j}>\epsilon_{k}}} \frac{1-t^{1+k-j}}{1-t^{k-j}}
$$

with $\epsilon_{j} \equiv \epsilon_{j}\left(J_{+}, J_{-}\right)$, and

$$
\begin{aligned}
U_{K, m}(\lambda):= & (-1)^{m} \sum_{\substack{I_{+}, I_{-} \subset K \\
I_{+} \cap I_{-}=\varnothing,\left|I_{+}\right|+\left|I_{-}\right|=m}}\left(\prod_{\substack{j \in I_{+} \\
\lambda_{j}=0}}\left(1-t_{0} t_{1} t^{n-j}\right)\left(1-t_{0} t_{2} t^{n-j}\right)\right. \\
& \times \prod_{\substack{j \in I_{-} \\
\lambda_{j}=1}}\left(1-t_{1} t_{2} t^{n-j}\right) \prod_{\substack{j, k \in K \\
\lambda_{j}=\lambda_{k}, \epsilon_{j}>\epsilon_{k}}} \frac{1-t^{1+k-j}}{1-t^{k-j}} \prod_{\substack{j \in I_{-}, k \in I_{+} \\
\lambda_{j}=\lambda_{k}+1}} \frac{1-t^{1+k-j}}{1-t^{k-j}} \\
& \left.\times \prod_{j \in K} t_{0}^{-\epsilon_{j}} \prod_{\substack{j, k \in K, j<k \\
\epsilon_{j} \neq \epsilon_{k}=0}} t^{-\epsilon_{j}} \prod_{\substack{j, k \in K, j<k \\
\lambda_{j}=\lambda_{k}, \epsilon_{k}-\epsilon_{j}=1}} t^{-1}\right),
\end{aligned}
$$

with $\epsilon_{j} \equiv \epsilon_{j}\left(I_{+}, I_{-}\right)$. Here we have employed the notation

$$
\epsilon_{j}\left(J_{+}, J_{-}\right):= \begin{cases}1 & \text { if } j \in J_{+} \\ -1 & \text { if } j \in J_{-} \\ 0 & \text { otherwise }\end{cases}
$$

for $J_{+}, J_{-} \subset\{1, \ldots, n\}$ with $J_{+} \cap J_{-}=\varnothing$, and we have also assumed the standard convention that empty products are equal to 1 .

When $l=1$, the action of $H_{l}(1)$ is relatively straightforward. Indeed, in this special case our operator amounts to a second-order difference operator of the form

$$
\left(H_{1} f\right)(\lambda)=u(\lambda) f(\lambda)+\sum_{\substack{1 \leq j \leq n \\ \lambda+e_{j} \in \Lambda}} w_{j}^{+}(\lambda) f\left(\lambda+e_{j}\right)+\sum_{\substack{1 \leq j \leq n \\ \lambda-e_{j} \in \Lambda}} w_{j}^{-}(\lambda) f\left(\lambda-e_{j}\right),
$$

with

$$
w_{j}^{+}(\lambda)=\left(\prod_{0 \leq r<s \leq 2}\left(1-t_{r} t_{s} t^{n-j}\right)\right)^{\delta_{\lambda_{j}}} \prod_{\substack{j<k \leq n \\ \lambda_{k}=\lambda_{j}}} \frac{1-t^{1+k-j}}{1-t^{k-j}}, \quad w_{j}^{-}(\lambda)=\prod_{\substack{1 \leq k<j \\ \lambda_{k}=\lambda_{j}}} \frac{1-t^{1+j-k}}{1-t^{j-k}}
$$

and

$$
u(\lambda)=-\sum_{\substack{1 \leq j \leq n \\ \lambda+e_{j} \in \Lambda}} t_{0}^{-1} t^{-(n-j)}\left(\left(1-t_{0} t_{1} t^{n-j}\right)\left(1-t_{0} t_{2} t^{n-j}\right)\right)^{\delta_{\lambda_{j}}} \prod_{\substack{j<k \leq n \\ \lambda_{k}=\lambda_{j}}} \frac{1-t^{1+k-j}}{1-t^{k-j}}
$$




$$
-\sum_{\substack{1 \leq j \leq n \\ \lambda-e_{j} \in \Lambda}} t_{0} t^{n-j}\left(1-t_{1} t_{2} t^{n-j}\right)^{\delta_{\lambda_{j}-1}} \prod_{\substack{1 \leq k<j \\ \lambda_{k}=\lambda_{j}}} \frac{1-t^{1+j-k}}{1-t^{j-k}},
$$

where $\delta_{m}:=1$ if $m=0$ and $\delta_{m}:=0$ if $m \neq 0$. Upon adding a harmless constant term of the form $\sum_{1 \leq j \leq n}\left(t_{0} t^{n-j}+t_{0}^{-1} t^{-(n-j)}\right)$ to the potential $u(\lambda)$, this reproduces the action of the semiinfinite $q$-boson Hamiltonian from [6, Section 4.1] restricted to the $n$-particle subspace (cf. also the proof of [6, Proposition 3]). The $q$-boson Hamiltonian considered in [8] amounts in turn to the parameter degeneration $t_{2} \rightarrow 0$ (cf. [6, Section 4.2]). In this interpretation the parts of $\lambda$ represent the positions of $n$ interacting quantum particles - dubbed $q$-bosons - that hop on a one-dimensional half-lattice formed by the nonnegative integers. The parameter $t$ corresponds to the deformation parameter $q$ of the underlying $q$-oscillator algebra and the parameters $t_{0}, t_{1}, t_{2}$ play the role of coupling constants for the boundary interaction on the lattice end-point at the origin (cf. Remark 2 at the end) [6, 8]. Here we will generally think of these parameters as rational indeterminates, unless explicitly understood otherwise.

The main result of this note is the following theorem, which states that the difference operators $H_{1}, \ldots, H_{n}$ constitute a system of commuting quantum integrals for the $n$-particle $q$-boson Hamiltonian $H=H_{1}(2)$.

Theorem 1. The difference operators $H_{1}, \ldots, H_{n}$ (1) mutually commute.

\section{Proof}

Our proof of Theorem 1 hinges on the $q \rightarrow 0$ degeneration of an explicit Pieri formula for the Macdonald-Koorwinder polynomials from [3, Section 6].

\subsection{Macdonald-Koornwinder polynomials at $q=0$}

In the limit $q \rightarrow 0$, the Macdonald-Koornwinder polynomial $[14,19]$ gives rise to a two-parameter extension of Macdonald's $B C$-type Hall-Littlewood function [18, $\S 10]$ of the form [27]

$$
P_{\lambda}(\xi)=c_{\lambda} \sum_{w \in W} C_{\lambda}(w \xi) e^{-i\langle\lambda, w \xi\rangle}, \quad \lambda \in \Lambda,
$$

with

$$
C_{\lambda}(\xi):=\prod_{1 \leq j<k \leq n} \frac{\left(1-t e^{i\left(\xi_{j}-\xi_{k}\right)}\right)\left(1-t e^{i\left(\xi_{j}+\xi_{k}\right)}\right)}{\left(1-e^{i\left(\xi_{j}-\xi_{k}\right)}\right)\left(1-e^{i\left(\xi_{j}+\xi_{k}\right)}\right)} \prod_{\substack{1 \leq j \leq n \\ \lambda_{j}>0}} \frac{\prod_{r=0}^{3}\left(1-t_{r} e^{i \xi_{j}}\right)}{1-e^{2 i \xi_{j}}}
$$

and

$$
c_{\lambda}:=\prod_{1 \leq j \leq n} \frac{t_{0}^{\lambda_{j}} t^{(n-j) \lambda_{j}}(1-t)}{\left(1-t^{j}\right)\left(1+t^{n-j}\right)^{\delta_{\lambda_{j}}} \prod_{1 \leq r \leq 3}\left(1-t_{0} t_{r} t^{n-j}\right)^{1-\delta_{\lambda_{j}}}} .
$$

Here $\langle\lambda, \xi\rangle \equiv \sum_{j=1}^{n} \lambda_{j} \xi_{j}$ and $W=S_{n} \ltimes\{-1,1\}^{n}$ denotes the hyperoctahedral group of signed permutations acting linearly on the components of $\xi=\left(\xi_{1}, \ldots, \xi_{n}\right) \in \mathbb{C}^{n}$. The trigonometric polynomial $P_{\lambda}(\xi)(3)$ is normalized so as to achieve a unit principal specialization value [6]:

$$
P_{\lambda}(i \rho)=1 \quad \text { at } \quad \rho=\sum_{1 \leq j \leq n}\left(\log \left(t_{0}\right)+(n-j) \log (t)\right) e_{j} .
$$


For parameter values in the domain

$$
t, t_{r} \in(-1,1) \backslash\{0\}, \quad r=0, \ldots, 3,
$$

the $q \rightarrow 0$ degeneration of the Macdonald-Koornwinder orthogonality ensures that $[14,27]$

$$
\int_{[0,2 \pi]^{n}} P_{\lambda}(\xi) \overline{P_{\mu}(\xi)}|\Delta(\xi)|^{2} \mathrm{~d} \xi=0 \quad \text { if } \quad \lambda \neq \mu
$$

where the orthogonality density is given by the squared modulus of

$$
\Delta(\xi)=\prod_{1 \leq j<k \leq n} \frac{\left(1-e^{i\left(\xi_{j}-\xi_{k}\right)}\right)\left(1-e^{i\left(\xi_{j}+\xi_{k}\right)}\right)}{\left(1-t e^{i\left(\xi_{j}-\xi_{k}\right)}\right)\left(1-t e^{i\left(\xi_{j}+\xi_{k}\right)}\right)} \prod_{1 \leq j \leq n} \frac{1-e^{2 i \xi_{j}}}{\prod_{r=0}^{3}\left(1-t_{r} e^{i \xi_{j}}\right)} .
$$

\subsection{Pieri formulas}

For a given choice of generators $E_{1}(\xi), \ldots, E_{n}(\xi)$ for the algebra of trigonometric polynomials with hyperoctahedral symmetry, the associated expansions of products of the form $E_{l}(\xi) P_{\lambda}(\xi)$ in the basis $P_{\mu}(\xi), \mu \in \Lambda$ give rise to a system of recurrence relations commonly referred to as Pieri formulas.

Theorem 2. For $l \in\{1, \ldots, n\}$, the hyperoctahedral Hall-Littlewood function $P_{\lambda}(\xi)$ (3) with $t_{3}=0$ satisfies the following Pieri-type recurrence relation:

$$
E_{l}(\xi) P_{\lambda}(\xi)=\sum_{\substack{J_{+}, J_{-} \subset\{1, \ldots, n\} \\ J_{+} \cap J_{-}=\varnothing,\left|J_{+}\right|+\left|J_{-}\right| \leq l \\ \lambda+e_{J_{+}}-e_{J_{-}} \in \Lambda}} U_{J_{+}^{c} \cap J_{-}^{c}, l-\left|J_{+}\right|-\left|J_{-}\right|}(\lambda) V_{J_{+}, J_{-}}(\lambda) P_{\lambda+e_{J_{+}}-e_{J_{-}}}(\xi),
$$

with

$$
\begin{aligned}
E_{l}(\xi):= & \sum_{1 \leq j_{1}<\cdots<j_{l} \leq n} \prod_{1 \leq k \leq l}\left(2 \cos \left(\xi_{j_{k}}\right)-t^{j_{k}-k} t_{0}-t^{-\left(j_{k}-k\right)} t_{0}^{-1}\right), \\
V_{J_{+}, J_{-}}(\lambda):= & \prod_{\substack{j \in J_{+} \\
\lambda_{j}=0}}\left(1-t_{0} t_{1} t^{n-j}\right)\left(1-t_{0} t_{2} t^{n-j}\right) \prod_{\substack{j \in J_{-} \\
\lambda_{j}=1}}\left(1-t_{1} t_{2} t^{n-j}\right) \\
& \times \prod_{\substack{1 \leq j<k \leq n \\
\lambda_{j}=\lambda_{k}, \epsilon_{j}>\epsilon_{k}}} \frac{1-t^{1+k-j}}{1-t^{k-j}} \prod_{1 \leq j \leq n} t_{0}^{-\epsilon_{j}} t^{-(n-j) \epsilon_{j}},
\end{aligned}
$$

where $\epsilon_{j} \equiv \epsilon_{j}\left(J_{+}, J_{-}\right)$, and with $U_{K, m}(\lambda)$ being defined as in Section 2 .

Proof. The stated formula boils down to a degeneration of the Pieri formula for the MacdonaldKoornwinder polynomials in [3, Theorem 6.1]. This degenerate formula is obtained through a straightforward but somewhat tedious computation that involves setting $t_{3}=q$ and performing the limit $q \rightarrow 0$. The present formulation moreover employs a compact expression for the multiplying polynomial $E_{l}(\xi)$ stemming from [13, equation (5.1)] (cf. also [9, Section 2]).

When $t_{2}=0$, Theorem 2 reduces to a Pieri formula for Macdonald's $B C$-type Hall-Littlewood functions. 


\subsection{Commutativity}

For $\lambda \in \Lambda$ such that $\lambda+e_{J_{+}}-e_{J_{-}} \in \Lambda$, we have the following functional relation between the coefficients of the difference operators in Theorem 1 and those of the Pieri formulas in Theorem 2:

$$
V_{J_{+}, J_{-}}(\lambda) h\left(\lambda+e_{J_{+}}-e_{J_{-}}\right)=W_{J_{+}, J_{-}}(\lambda) h(\lambda)
$$

with

$$
h(\lambda):=\prod_{\substack{1 \leq j \leq n \\ \lambda_{j}>0}} t_{0}^{\lambda_{j}} t^{(n-j) \lambda_{j}}\left(1-t_{1} t_{2} t^{n-j}\right) .
$$

The upshot is that upon conjugating $H_{l}$ (1) with the (invertible) multiplication operator in $C(\Lambda)$ of the form $f \rightarrow h f$, the coefficients $W_{J_{+}, J_{-}}(\lambda)$ get replaced by the Pieri coefficients $V_{J_{+}, J_{-}}(\lambda)$. The Pieri formula in Theorem 2 tells us that the resulting conjugated difference operators commute on the joint eigenbasis of hyperoctahedral Hall-Littlewood functions (3) (viewed as lattice functions of $\lambda \in \Lambda$ depending on a polynomial spectral parameter $\xi \in \mathbb{R}^{n}$ ). Indeed, from this perspective the Pieri formula corresponds to an eigenvalue equation with the bounded function $E_{l}(\xi)$ playing the role of the eigenvalue. The orthogonality relations (4) moreover guarantee the completeness of these (generalized) eigenfunctions (cf. Remark 1 below), i.e., the difference operators in question commute in fact as bounded operators in the Hilbert space $\ell^{2}(\Lambda, \nu) \subset C(\Lambda)$ determined by a discrete measure with weights

$$
\nu_{\lambda}=\left(\int_{[0,2 \pi]^{n}}\left|P_{\lambda}(\xi) \Delta(\xi)\right|^{2} \mathrm{~d} \xi\right)^{-1}, \quad \lambda \in \Lambda .
$$

This means that the operators commute in particular on the (stable) subspace of $C(\Lambda)$ consisting of the lattice functions with finite support. But then the commutativity must actually hold on the whole space $C(\Lambda)$, as given any $f \in C(\Lambda)$ and any $\lambda \in \Lambda$, the evaluation at $\lambda$ of the commutator of two of such difference operators acting on $f$ depends manifestly only on evaluations of $f$ at a finite number of lattice points in $\Lambda$. Finally, the commutativity is extended beyond the parameter values in the orthogonality domain by analyticity.

Remark 1. Notice that away from the boundary (i.e., for $\lambda_{1} \geq \cdots \geq \lambda_{n}>0$ ) the wave function $P_{\lambda}(\xi)$ (3) decomposes as a linear combination of plane waves (of Bethe Ansatz form). In particular, the wave function in question does not belong to the Hilbert space $\ell^{2}(\Lambda, \nu)$ and constitutes in fact a generalized eigenfunction of the discrete difference operators arising from the Pieri formulas. (The spectra of these bounded difference operators are absolutely continuous rather than discrete.) The orthogonality relations (4) do nevertheless imply that any $f$ in $\ell^{2}(\Lambda, \nu)$ can be represented through a wave packet via the associated (generalized) Fourier transform:

$$
f(\lambda)=\int_{[0,2 \pi]^{n}} \hat{f}(\xi) P_{\lambda}(\xi)|\Delta(\xi)|^{2} \mathrm{~d} \xi, \quad \lambda \in \Lambda,
$$

where

$$
\hat{f}(\xi)=\sum_{\lambda \in \Lambda} f(\lambda) \overline{P_{\lambda}(\xi)} \nu_{\lambda}
$$

which confirms the completeness of our generalized eigenfunctions. (Here the convergence of the sum on the r.h.s. of (5) is in the strong $L^{2}\left([0,2 \pi]^{n},|\Delta(\xi)|^{2} \mathrm{~d} \xi\right)$ Hilbert space sense.) 


\section{Extension to four-parameter boundary interactions}

In principle there is no genuine obstruction preventing us from adapting the commuting quantum integrals of Theorem 1 to the case of the more general semi-infinite $q$-boson system in [6] with four-parameter boundary interactions. For this purpose, one needs to establish a generalization of the Pieri formula in Theorem 2 covering the hyperoctahedral Hall-Littlewood polynomials $P_{\lambda}(\xi)(3)$ with $t_{3}$ arbitrary. It turns out, however, that in this more general setting the coefficients of the Pieri formulas (and thus also those of the corresponding quantum integrals) become quite baroque. We wrap up by indicating briefly how the above formulas are to be modified when dealing with such general boundary interactions involving four parameters $t_{0}, \ldots, t_{3}$.

\subsection{Pieri coefficients}

Even though the global structure of the Pieri formula for the hyperoctahedral Hall-Littlewood functions $P_{\lambda}(\xi)$ (3) remains of the form described by Theorem 2 when dropping the condition that $t_{3}$ be zero, the fine structure of the coefficients is now more intricate:

$$
\begin{aligned}
V_{J_{+}, J_{-}}(\lambda)= & \prod_{\substack{j \in J_{+} \\
\lambda_{j}=0}} \frac{\left(1-\tau t^{n-j+m_{0}(\lambda)+m_{1}(\lambda)-m_{1}^{+}(\lambda)}\right) \prod_{1 \leq r \leq 3}\left(1-t_{0} t_{r} t^{n-j}\right)}{\left(1-\tau t^{2(n-j)}\right)\left(1-\tau t^{2(n-j)+1}\right)} \\
& \times \prod_{\substack{j \in J_{+} \\
\lambda_{j}=1}}\left(1-\tau t^{n-j+m_{0}(\lambda)}\right) \prod_{\substack{j \in J_{-} \\
\lambda_{j}=1}} \frac{\left(1-\tau t^{n-j-1}\right) \prod_{1 \leq r<s \leq 3}\left(1-t_{r} t_{s} t^{n-j}\right)}{\left(1-\tau t^{2(n-j)}\right)\left(1-\tau t^{2(n-j)-1}\right)} \\
& \times \prod_{\substack{1 \leq j<k \leq n \\
\lambda_{j}=\lambda_{k}, \epsilon_{j}>\epsilon_{k}}} \frac{1-t^{1+k-j}}{1-t^{k-j}} \prod_{1 \leq j \leq n} t_{0}^{-\epsilon_{j}} t^{-(n-j) \epsilon_{j}}
\end{aligned}
$$

and

$$
\begin{aligned}
& U_{K, m}(\lambda)=(-1)^{m} \sum_{\substack{I_{+}, I_{-} \subset K \\
I_{+} \cap I_{-}=\varnothing,\left|I_{+}\right|+\left|I_{-}\right|=m}}\left(\prod_{\substack{j \in I_{+} \\
\lambda_{j}=0}} \frac{\prod_{1 \leq r \leq 3}\left(1-t_{0} t_{r} t^{n-j}\right)}{1-\tau t^{2(n-j)}} \prod_{\substack{j \in I_{+} \\
\lambda_{j}=1}}\left(1-\tau t^{n-j}\right)\right. \\
& \times \prod_{\substack{j \in I_{-} \\
\lambda_{j}=1}} \frac{\prod_{1 \leq r<s \leq 3}\left(1-t_{r} t_{s} t^{n-j}\right)}{1-\tau t^{2(n-j)}} \prod_{\substack{j, k \in K, j<k \\
\epsilon_{j}+\epsilon_{k} \in\{-2,1,2\} \\
\lambda_{j}=1, \lambda_{k}=\delta_{1+\epsilon_{k}}}} \frac{1-\tau t^{2 n+1-j-k}}{1-\tau t^{2 n-j-k}} \\
& \times \prod_{\substack{j \in I_{+} \cup I_{-}, k \in K \backslash I_{-} \\
j<k, \epsilon_{k}-\epsilon_{j} \in\{0,1\} \\
\lambda_{j}=\delta_{1+\epsilon_{j}}, \lambda_{k}=0}} \frac{1-\tau t^{2 n-1-j-k}}{1-\tau t^{2 n-j-k}} \prod_{\substack{j, k \in K \\
\lambda_{j}=\lambda_{k}, \epsilon_{j}>\epsilon_{k}}} \frac{1-t^{1+k-j}}{1-t^{k-j}} \prod_{\substack{j \in I_{-}, k \in I_{+} \\
\lambda_{j}=\lambda_{k}+1}} \frac{1-t^{1+k-j}}{1-t^{k-j}} \\
& \times \prod_{j \in K} t_{0}^{-\epsilon_{j}} \prod_{\substack{j, k \in K, j<k \\
\epsilon_{j} \neq \epsilon_{k}=0}} t^{-\epsilon_{j}} \prod_{\substack{j, k \in K, j<k \\
\lambda_{j}=\lambda_{k}, \epsilon_{k}-\epsilon_{j}=1}} t^{-1},
\end{aligned}
$$

where $m_{l}(\lambda)=\left|\left\{1 \leq j \leq n \mid \lambda_{j}=l\right\}\right|, m_{l}^{+}(\lambda)=\left|\left\{j \in J_{+} \mid \lambda_{j}=l\right\}\right|$, and $\tau=t_{0} t_{1} t_{2} t_{3}$. These Pieri coefficients are obtained from [3, Theorem 6.1] in the limit $q \rightarrow 0$. 


\section{2 $q$-Boson quantum integrals}

By virtue of the argumentation in Section 3.3, the Pieri formulas at issue give rise to commuting difference operators $H_{1}, \ldots, H_{n}$ of the form stated in equation (1) with

$$
\begin{aligned}
W_{J_{+}, J_{-}}(\lambda)= & \prod_{\substack{j \in J_{+} \\
\lambda_{j}=1}}\left(1-\tau t^{n-j+m_{0}(\lambda)}\right) \prod_{\substack{1 \leq j<k \leq n \\
\lambda_{j}=\lambda_{k}, \epsilon_{j}>\epsilon_{k}}} \frac{1-t^{1+k-j}}{1-t^{k-j}} \\
& \times \prod_{\substack{j \in J_{+} \\
\lambda_{j}=0}} \frac{\left(1-\tau t^{n-j-1}\right)\left(1-\tau t^{n-j+m_{0}(\lambda)+m_{1}(\lambda)-m_{1}^{+}(\lambda)}\right) \prod_{0 \leq r<s \leq 3}\left(1-t_{r} t_{s} t^{n-j}\right)}{\left(1-\tau t^{2(n-j)-1}\right)\left(1-\tau t^{2(n-j)}\right)^{2}\left(1-\tau t^{2(n-j)+1}\right)}
\end{aligned}
$$

and with $U_{K, m}(\lambda)$ taken from equation (6). The relation between the coefficients of the Pieri formula and those of the difference operators is again governed by a functional identity of the type in Section 3.3 with

$$
h(\lambda)=\prod_{1 \leq j \leq n} t_{0}^{\lambda_{j}} t^{(n-j) \lambda_{j}}\left(1-\tau t^{n+m_{0}(\lambda)-j-1}\right)^{\delta_{\lambda_{j}}} \prod_{1 \leq r<s \leq 3}\left(1-t_{r} t_{s} t^{n-j}\right)^{1-\delta_{\lambda_{j}}} .
$$

When $l=1$ the corresponding difference operator $H_{l}$ now reduces to $H_{1}(2)$ with

$$
\begin{aligned}
w_{j}^{+}(\lambda)= & \left(1-\tau t^{2 m_{0}(\lambda)+m_{1}(\lambda)-1}\right)^{\delta_{\lambda_{j}-1}+\delta_{\lambda_{j}}} \prod_{\substack{j<k \leq n \\
\lambda_{k}=\lambda_{j}}} \frac{1-t^{1+k-j}}{1-t^{k-j}} \\
& \times\left(\frac{\left(1-\tau t^{n-j-1}\right) \prod_{\substack{0 \leq r<s \leq 3 \\
\left(1-\tau t^{2(n-j)-1}\right)\left(1-\tau t^{2(n-j)}\right)^{2}\left(1-\tau t^{2(n-j)+1}\right)}}\left(1-t_{s} t^{n-j}\right)}{(1-},\right. \\
w_{j}^{-}(\lambda)= & \prod_{\substack{1 \leq k<j \\
\lambda_{k}=\lambda_{j}}} \frac{1-t^{1+j-k}}{1-t^{j-k}},
\end{aligned}
$$

and

$$
\begin{aligned}
u(\lambda)=- & \sum_{\substack{1 \leq j \leq n \\
\lambda+e_{j} \in \Lambda}} t_{0}^{-1} t^{-(n-j)}\left(1-\tau t^{2 m_{0}(\lambda)+m_{1}(\lambda)-1}\right)^{\delta_{\lambda_{j}-1}+\delta_{\lambda_{j}}} \\
& \times\left(\frac{\prod_{1 \leq r \leq 3}\left(1-t_{0} t_{r} t^{n-j}\right)}{\left(1-\tau t^{2(n-j)}\right)\left(1-\tau t^{2(n-j)+1}\right)}\right)^{\delta_{\lambda_{j}}} \prod_{\substack{j<k \leq n \\
\lambda_{k}=\lambda_{j}}} \frac{1-t^{1+k-j}}{1-t^{k-j}} \\
& -\sum_{\substack{1 \leq j \leq n \\
\lambda-e_{j} \in \Lambda}} t_{0} t^{n-j}\left(\frac{\left(1-\tau t^{n-j-1}\right) \prod_{1 \leq r<s \leq 3}\left(1-t_{r} t_{s} t^{n-j}\right)}{\left(1-\tau t^{2(n-j)-1}\right)\left(1-\tau t^{2(n-j)}\right)}\right)^{\delta_{\lambda_{j}-1}} \prod_{\substack{1 \leq k<j \\
\lambda_{k}=\lambda_{j}}} \frac{1-t^{1+j-k}}{1-t^{j-k}} .
\end{aligned}
$$

Upon adding the constant term $\sum_{1 \leq j \leq n}\left(t_{0} t^{n-j}+t_{0}^{-1} t^{-(n-j)}\right)$, this reproduces the $n$-particle $q$-boson Hamiltonian of [6, Section 3].

Remark 2. In [6] a Fock space description of the particle Hamiltonian $H_{1}(2)$, (7a)-(7c) was provided as a system of $q$-bosons on the nonnegative integer lattice perturbed at the lattice-end. 
Specifically, the boundary interactions arise in this picture from a deformation of the $q$-boson field algebra at the origin and its nearest neighboring lattice point parametrized by $t_{0}, t_{1}, t_{2}, t_{3}$. In general, i.e., when $\tau=t_{0} t_{1} t_{2} t_{3} \neq 0$, the deformed $q$-boson field algebra is no longer ultralocal at the boundary as the commutativity between the creation and annihilation operators at the origin and its nearest neighboring site is lost. When $t_{3}=0$, the deformation of the $q$-boson field algebra is restricted only to the lattice end-point at the origin and the ultralocality is restored [6, Section 4.1]. When both $t_{2}=t_{3}=0$, the boundary interaction degenerates and decomposes into an interaction arising from a one-parameter deformation of the $q$-boson field algebra and a one-parameter additive potential term of the Hamiltonian, supported at the lattice end-point [8].

\section{Acknowledgements}

This work was supported in part by the Fondo Nacional de Desarrollo Científico y Tecnológico (FONDECYT) Grants \# 1130226 and \# 1141114.

\section{References}

[1] Bogoliubov N.M., Izergin A.G., Kitanine N.A., Correlation functions for a strongly correlated boson system, Nuclear Phys. B 516 (1998), 501-528, solv-int/9710002.

[2] Borodin A., Corwin I., Petrov L., Sasamoto T., Spectral theory for interacting particle systems solvable by coordinate Bethe ansatz, arXiv:1407.8534.

[3] van Diejen J.F., Properties of some families of hypergeometric orthogonal polynomials in several variables, Trans. Amer. Math. Soc. 351 (1999), 233-270, q-alg/9604004.

[4] van Diejen J.F., Scattering theory of discrete (pseudo) Laplacians on a Weyl chamber, Amer. J. Math. 127 (2005), 421-458, math-ph/0412097.

[5] van Diejen J.F., Diagonalization of an integrable discretization of the repulsive delta Bose gas on the circle, Comm. Math. Phys. 267 (2006), 451-476, math-ph/0604029.

[6] van Diejen J.F., Emsiz E., Boundary interactions for the semi-infinite $q$-boson system and hyperoctahedral Hall-Littlewood polynomials, SIGMA 9 (2013), 077, 12 pages, arXiv:1312.1028.

[7] van Diejen J.F., Emsiz E., Diagonalization of the infinite $q$-boson system, J. Funct. Anal. 266 (2014), 5801-5817, arXiv:1308.2237.

[8] van Diejen J.F., Emsiz E., The semi-infinite $q$-boson system with boundary interaction, Lett. Math. Phys. 104 (2014), 103-113, arXiv:1308.2242.

[9] van Diejen J.F., Emsiz E., Branching formula for Macdonald-Koornwinder polynomials, arXiv:1408.2280.

[10] van Diejen J.F., Vinet L., The quantum dynamics of the compactified trigonometric Ruijsenaars-Schneider model, Comm. Math. Phys. 197 (1998), 33-74, math/9709221.

[11] Dorlas T.C., Orthogonality and completeness of the Bethe ansatz eigenstates of the nonlinear Schroedinger model, Comm. Math. Phys. 154 (1993), 347-376.

[12] Klimyk A., Schmüdgen K., Quantum groups and their representations, Texts and Monographs in Physics, Springer-Verlag, Berlin, 1997.

[13] Komori Y., Noumi M., Shiraishi J., Kernel functions for difference operators of Ruijsenaars type and their applications, SIGMA 5 (2009), 054, 40 pages, arXiv:0812.0279.

[14] Koornwinder T.H., Askey-Wilson polynomials for root systems of type $B C$, in Hypergeometric Functions on Domains of Positivity, Jack Polynomials, and Applications (Tampa, FL, 1991), Contemp. Math., Vol. 138, Amer. Math. Soc., Providence, RI, 1992, 189-204.

[15] Korepin V.E., Bogoliubov N.M., Izergin A.G., Quantum inverse scattering method and correlation functions, Cambridge Monographs on Mathematical Physics, Cambridge University Press, Cambridge, 1993.

[16] Korff C., Cylindric versions of specialised Macdonald functions and a deformed Verlinde algebra, Comm. Math. Phys. 318 (2013), 173-246, arXiv:1110.6356.

[17] Lieb E.H., Liniger W., Exact analysis of an interacting Bose gas. I. The general solution and the ground state, Phys. Rev. 130 (1963), 1605-1616. 
[18] Macdonald I.G., Orthogonal polynomials associated with root systems, Sém. Lothar. Combin. 45 (2000), Art. B45a, 40 pages, math.QA/0011046.

[19] Macdonald I.G., Affine Hecke algebras and orthogonal polynomials, Cambridge Tracts in Mathematics, Vol. 157, Cambridge University Press, Cambridge, 2003.

[20] Majid S., Foundations of quantum group theory, Cambridge University Press, Cambridge, 1995.

[21] Povolotsky A.M., On the integrability of zero-range chipping models with factorized steady states, J. Phys. A: Math. Theor. 46 (2013), 465205, 25 pages, arXiv:1308.3250.

[22] Ruijsenaars S.N.M., Finite-dimensional soliton systems, in Integrable and Superintegrable Systems, World Sci. Publ., Teaneck, NJ, 1990, 165-206.

[23] Ruijsenaars S.N.M., Factorized weight functions vs. factorized scattering, Comm. Math. Phys. 228 (2002), 467-494.

[24] Sasamoto T., Wadati M., Exact results for one-dimensional totally asymmetric diffusion models, J. Phys. A: Math. Gen. 31 (1998), 6057-6071.

[25] Takeyama Y., A deformation of affine Hecke algebra and integrable stochastic particle system, J. Phys. A: Math. Theor. 47 (2014), 465203, 19 pages, arXiv:1407.1960.

[26] Tsilevich N.V., The quantum inverse scattering problem method for the $q$-boson model, and symmetric functions, Funct. Anal. Appl. 40 (2006), 207-217, math-ph/0510073.

[27] Venkateswaran V., Symmetric and nonsymmetric Hall-Littlewood polynomials of type BC, arXiv:1209.2933. 